\title{
On existence and uniqueness of positive solutions to a class of fractional boundary value problems
}

\author{
J Caballero*, J Harjani and K Sadarangani
}

*Correspondence: fefi@dma.ulpgc

Departamento de Matemáticas, Universidad de Las Palmas de Gran Canaria, Campus de Tafira Baja, 35017 Las Palmas de Gran Canaria, Spain

\begin{abstract}
The purpose of this paper is to investigate the existence and uniqueness of positive solutions for the following fractional boundary value problem

$$
\begin{aligned}
& D_{0^{+}}^{\alpha} u(t)+f(t, u(t))=0, \quad 0<t<1, \\
& u(0)=u(1)=u^{\prime}(0)=0,
\end{aligned}
$$

where $2<\alpha \leq 3$ and $D_{0^{+}}^{\alpha}$ is the Riemann-Liouville fractional derivative.

Our analysis relies on a fixed-point theorem in partially ordered metric spaces. The autonomous case of this problem was studied in the paper [Zhao et al., Abs. Appl. Anal., to appear], but in Zhao et al. (to appear), the question of uniqueness of the solution is not treated.

We also present some examples where we compare our results with the ones obtained in Zhao et al. (to appear).

2010 Mathematics Subject Classification: 34B15
\end{abstract}

Keywords: fractional boundary value problem, fixed-point theorem, positive solution

\section{Introduction}

Differential equations of fractional order occur more frequently on different research areas and engineering such as physics, chemistry, economics, etc. Indeed, we can find numerous applications in viscoelasticity, electrochemistry control, porous media, electromagnetic, etc. [1-6].

For an extensive collection of results about this type of equations, we refer the reader to the monograph by Kilbas and Trujillo [7], Samko, Kilbas, and Marichev [8], Miller and Ross [9], and Podlubny [10].

On the other hand, some basic theory for the initial value problems of fractional differential equations involving the Riemann-Lioville differential operator has been discussed by Lakshmikantham et al. [11,12], Bai et al. [13-16], Zhang [17], etc.

In [15], the authors studied the following two-point boundary value problem of fractional order

$$
\begin{aligned}
& D_{0^{+}}^{\alpha} u(t)+a(t) f(t, u(t))=0, \quad 0<t<1,1<\alpha \leq 2 \\
& u(0)=u(1)=0,
\end{aligned}
$$

\section{空


and they proved the existence of positive solutions by means of the Krasnosel'skii fixed-point theorem and Legget-Williams fixed-point theorem.

Recently, in the paper [18] to appear in this special issue, the authors studied the existence of positive solutions for the following autonomous boundary value problem of fractional order

$$
\begin{aligned}
& D_{0^{+}}^{\alpha} u(t)+\lambda f(u(t))=0, \quad 0<t<1, \\
& u(0)=u(1)=u^{\prime}(0)=0,
\end{aligned}
$$

where $2<\alpha \leq 3, \lambda$ is a positive parameter, and $f:(0, \infty) \rightarrow(0, \infty)$ is continuous.

Motivated by this last work, in this paper, we discuss the existence and uniqueness of positive solutions for the nonautonomous version of Problem (1). More precisely, we study the following problem

$$
\begin{aligned}
& D_{0^{+}}^{\alpha} u(t)+f(t, u(t))=0, \quad 0<t<1, \\
& u(0)=u(1)=u^{\prime}(0)=0,
\end{aligned}
$$

where $2<\alpha \leq 3$ and $f:[0,1] \times[0, \infty) \rightarrow[0, \infty)$ is a continuous function.

Notice that in [18] the question of uniqueness of solutions is not treated.

In our study, the main tool is a fixed-point theorem in partially ordered sets, which gives us uniqueness of the solution. This result appears in [19].

\section{Basic facts}

For the convenience of the reader, we present some definitions, lemmas, and basic results that will be used later.

Definition 1. [7] The Riemann-Liouville fractional derivative of order $\alpha>0$ of a function $f:(0, \infty) \rightarrow \mathbb{R}$ is given by

$$
D_{0^{+}}^{\alpha} f(t)=\frac{1}{\Gamma(n-\alpha)}\left(\frac{d}{d t}\right)^{n} \int_{0}^{t} \frac{f(s)}{(t-s)^{\alpha-n+1}} \mathrm{~d} s,
$$

where $n=[\alpha]+1$ and $[\alpha]$ denotes the integer part of $\alpha$ and $\Gamma(\alpha)$ denotes the gamma function, provided that the right side is pointwise defined on $(0, \infty)$.

Definition 2. [7] The Riemman-Liouville fractional integral of order $\alpha>0$ of a function $f:(0, \infty) \rightarrow \mathbb{R}$ is defined by

$$
I_{0^{+}}^{\alpha} f(t)=\frac{1}{\Gamma(\alpha)} \int_{0}^{t}(t-s)^{\alpha-1} f(s) \mathrm{d} s,
$$

provided that the right side is pointwise defined on $(0, \infty)$.

The following two lemmas can be found in [20].

Lemma 1. Let $\alpha>0$ and $u \in C(0,1) \cap L^{1}(0,1)$. Then, the fractional differential equation

$$
D_{0^{+}}^{\alpha} u(t)=0
$$

has

$$
u(t)=c_{1} t^{\alpha-1}+c_{2} t^{\alpha-2}+\cdots+c_{n} t^{\alpha-n},
$$

where $c_{i} \in \mathbb{R}(i=1, \ldots n)$ and $n=[\alpha]+1$ as unique solution. 
Lemma 2. Assume that $u \in C(0,1) \cap L^{1}(0,1)$ with a fractional derivative of order $\alpha$ $>0$ that belongs to $C(0,1) \cap L^{1}(0,1)$. Then,

$$
I_{0^{+}}^{\alpha} D_{0^{+}}^{\alpha} u(t)=u(t)+c_{1} t^{\alpha-1}+c_{2} t^{\alpha-2}+\cdots+c_{n} t^{\alpha-n},
$$

for some $c_{i} \in \mathbb{R}(i=1, \ldots, n)$ and $n=[\alpha]+1$.

In [21], it is proved the following result by using Lemmas 1 and 2 .

Lemma 3. Given $f \in C[0,1]$ and $2<\alpha \leq 3$. The unique solution of

$$
\begin{aligned}
& D_{0^{+}}^{\alpha} u(t)+f(t)=0, \quad 0<t<1, \\
& u(0)=u(1)=u^{\prime}(0)=0,
\end{aligned}
$$

is

$$
u(t)=\int_{0}^{1} G(t, s) f(s) \mathrm{d} s
$$

where

$$
G(t, s)= \begin{cases}\frac{t^{\alpha-1}(1-s)^{\alpha-1}-(t-s)^{\alpha-1}}{\Gamma(\alpha)}, & 0 \leq s \leq t \leq 1 \\ \frac{t^{\alpha-1}(1-s)^{\alpha-1}}{\Gamma(\alpha)}, & 0 \leq t \leq s \leq 1 .\end{cases}
$$

is the Green's function associated to the boundary value problem (3).

Remark 1. In [21], it is proved that $G(t, s) \geq 0$, for $t, s \in[0,1]$.

Now, we present the fixed-point theorems that we will use later. These results appear in [19].

Theorem 1. Let $(X, \leq)$ be a partially ordered set and suppose that there exists a metric $d$ in $X$ such that $(X, d)$ is a complete metric space. Assume that $X$ satisfies the following condition

$$
\text { if }\left(x_{n}\right) \text { is a nondecreasing sequence in } X \text { such that } x_{n} \rightarrow x \text { then } x_{n} \leq x \text {, for all } n \in \mathbb{N} \text {. }
$$

Let $T: X \rightarrow X$ be a nondecreasing mapping such that

$$
d(T x, T y) \leq d(x, y)-\psi(d(x, y)), \quad \text { for } x \geq y,
$$

where $\psi:[0, \infty) \rightarrow[0, \infty)$ is a continuous and nondecreasing function such that $\psi$ is positive in $(0, \infty), \psi(0)=0$, and $\lim _{t \rightarrow \infty} \psi(t)=\infty$. If there exists $x_{0} \in X$ with $x_{0} \leq T x_{0}$ then $T$ has a fixed point.

Moreover, if $(X, \leq)$ satisfies the following condition:

for each $x, y \in X$ there exists $z \in X$ which is comparable to $\mathrm{x}$ and $\mathrm{y}$,

we have the following result.

Theorem 2. Adding condition (5) to the hypotheses of Theorem 1, we obtain uniqueness of the fixed point.

Remark 2. In Theorems 1 and 2, the condition $\lim _{t \rightarrow \infty} \psi(t)=\infty$ is superfluous.

In our considerations, we will work in the Banach space $C[0,1]=\{x:[0,1] \rightarrow \mathbb{R}$, continuous $\}$ with the standard distance given by $d(x, y)=\sup _{0 \leq t \leq 1}\{|x(t)-y(t)|\}$.

Moreover, this space can be equipped with a partial order given by

$$
x, y \in C[0,1], \quad x \leq y \Leftrightarrow x(t) \leq y(t), \quad \text { for } t \in[0,1] .
$$


In [22], it is proved that $(C[0,1], \leq)$ with the above-mentioned metric satisfies condition (4) of Theorem 1. Moreover, for $x, y \in C[0,1]$, as the function $\max (x, y) \in C[0$, $1],(C[0,1], \leq)$ satisfies condition (5).

By $\mathcal{F}$, we denote the class of functions $\psi:[0, \infty) \rightarrow[0, \infty)$ continuous, nondecreasing, positive in $(0, \infty)$ and $\psi(0)=0$, and by $\mathcal{J}$ the class of functions $\phi:[0, \infty) \rightarrow[0, \infty)$ continuous, nondecreasing, and satisfying that $I-\varphi \in \mathcal{F}$, where $I$ denotes the identity mapping on $[0, \infty)$.

\section{Main result}

Our starting point of this section is the following result about Green's function appearing in Section 2.

Lemma 4. $\max _{t \in[0,1]} \int_{0}^{1} G(t, s) d s=\frac{1}{\Gamma(\alpha+1)}\left[\left(\frac{\alpha-1}{\alpha}\right)^{\alpha-1}-\left(\frac{\alpha-1}{\alpha}\right)\right]$

Proof. In fact,

$$
\begin{aligned}
\int_{0}^{1} G(t, s) \mathrm{d} s & =\int_{0}^{t} G(t, s) \mathrm{d} s+\int_{t}^{1} G(t, s) \mathrm{d} s \\
& =\int_{0}^{t} \frac{t^{\alpha-1}(1-s)^{\alpha-1}-(t-s)^{\alpha-1}}{\Gamma(\alpha)} \mathrm{d} s+\int_{t}^{1} \frac{t^{\alpha-1}(1-s)^{\alpha-1}}{\Gamma(\alpha)} \mathrm{d} s \\
& =\int_{0}^{1} \frac{t^{\alpha-1}(1-s)^{\alpha-1}}{\Gamma(\alpha)} \mathrm{d} s-\int_{0}^{t} \frac{(t-s)^{\alpha-1}}{\Gamma(\alpha)} \mathrm{d} s \\
& =\frac{1}{\Gamma(\alpha)}\left[\frac{t^{\alpha-1}}{\alpha}-\frac{t^{\alpha}}{\alpha}\right]=\frac{1}{\Gamma(\alpha+1)}\left(t^{\alpha-1}-t^{\alpha}\right)
\end{aligned}
$$

By an elemental calculation, it can be proved that the maximum of $h(t)=\int_{0}^{1} G(t, s) \mathrm{d} s=\frac{1}{\Gamma(\alpha+1)}\left(t^{\alpha-1}-t^{\alpha}\right)$ is reached at $t_{0}=\frac{\alpha-1}{\alpha}$, thus,

$$
\max _{0 \leq t \leq 1} \int_{0}^{1} G(t, s) \mathrm{d} s=\frac{1}{\Gamma(\alpha+1)}\left[\left(\frac{\alpha-1}{\alpha}\right)^{\alpha-1}-\left(\frac{\alpha-1}{\alpha}\right)^{\alpha}\right]
$$

$\square$

In the sequel, we present the main result of this paper.

For convenience, we put $A=\frac{1}{\Gamma(\alpha+1)}\left[\left(\frac{\alpha-1}{\alpha}\right)^{\alpha-1}-\left(\frac{\alpha-1}{\alpha}\right)^{\alpha}\right]$.

Theorem 3. Our Problem (2) has a unique nonnegative solution $u(t)$ if the following conditions are satisfied:

(H1) $f:[0,1] \times[0, \infty) \rightarrow[0, \infty)$ is continuous and nondecreasing respect to the second argument.

(H2) There exists $0<\lambda \leq \frac{1}{A}$ such that, for $x, y \in[0, \infty)$ with $y \geq x$ and $t \in[0,1]$,

$$
f(t, y)-f(t, x) \leq \lambda \varphi(y-x),
$$

where $\varphi \in \mathcal{J}$.

Proof. Consider the cone

$$
P=\{u \in C[0,1]: u(t) \geq 0\} .
$$


Obviously, $(P, d)$ with $d(x, y)=\sup \{|x(t)-y(t)|: t \in[0,1]\}$ is a complete metric space satisfying conditions (4) and (5).

Consider the operator defined by

$$
(T x)(t)=\int_{0}^{1} G(t, s) f(s, x(s)) \mathrm{d} s, \quad \text { for } x \in P,
$$

where $G(t, s)$ is the Green's function appearing in Section 2. Obviously, $T$ applies $P$ into itself since $f(t, x)$ and $G(t, s)$ are nonnegative continuous functions.

In what follows we check that assumptions in Theorem 2 are satisfied.

Firstly, the operator $T$ is nondecreasing.

Indeed, by (H1), for $u, v \in P, u \geq v$, and $t \in[0,1]$, we have

$$
(T u)(t)=\int_{0}^{1} G(t, s) f(s, u(s)) \mathrm{d} s \geq \int_{0}^{1} G(t, s) f(s, v(s)) \mathrm{d} s=(T v)(t) .
$$

Now, we prove that $T$ satisfies the contractive condition appearing in Theorem 1 .

In fact, for $u, v \in P$ and $u \geq v$ and, taking into account assumption (H2), we get

$$
\begin{aligned}
\mathrm{d}(T u, T v) & =\sup _{t \in[0,1]}\{|T u(t)-T v(t)|\} \\
& =\sup _{t \in[0,1]}\{(T u(t)-T v(t))\} \\
& =\sup _{t \in[0,1]} \int_{0}^{1} G(t, s)(f(s, u(s))-f(s, v(s))) \mathrm{d} s \\
& \leq \sup _{t \in[0,1]} \int_{0}^{1} G(t, s) \lambda \varphi(u(s)-v(s)) \mathrm{d} s .
\end{aligned}
$$

As $\varphi \in \mathcal{J}, \phi$ is nondecreasing, and, taking into account (H2) and Lemma 4, we obtain

$$
\begin{aligned}
\mathrm{d}(T u, T v) & \leq \lambda \varphi(\mathrm{d}(u, v)) \cdot \sup _{t \in[0,1]} \int_{0}^{1} G(t, s) \mathrm{d} s \\
& =\lambda \varphi(\mathrm{d}(u, v)) \cdot A \leq \varphi(\mathrm{d}(u, v))=\mathrm{d}(u, v)-(\mathrm{d}(u, v)-\varphi(\mathrm{d}(u, v))) .
\end{aligned}
$$

Put $\psi(x)=x-\phi(x)$. As $\varphi \in \mathcal{J}$, this means that $\psi \in \mathcal{F}$ and from the last inequality

$$
\mathrm{d}(T u, T v) \leq \mathrm{d}(u, v)-\psi(\mathrm{d}(u, v)) .
$$

This proves that $T$ satisfies the contractive condition of Theorem 1 .

Finally, the nonnegative character of the function $G(t, s)$ and $f(t, x)$ [assumption (H1)] gives us

$$
(T 0)(t)=\int_{0}^{1} G(t, s) f(s, 0) \mathrm{d} s \geq 0,
$$

where 0 denotes the zero function.

Therefore, Theorem 2 says us that Problem (2) has a unique nonnegative solution.

口 
In the sequel, we present a sufficient condition for the existence and uniqueness of positive solutions for Problem (2) (positive solution means $x(t)>0$ for $t \in(0,1)$ ). The proof of this condition is similar to the proof of Theorem 2.3 of [23]. We present this proof for completeness.

Theorem 4. Under assumptions of Theorem 3 and suppose that $f\left(t_{0}, 0\right) \neq 0$ for certain $t_{0} \in[0,1]$. Then, Problem (2) has a unique positive solution.

Proof. Consider the nonnegative solution $x(t)$ for Problem (2) whose existence is guaranteed by Theorem 3 .

In the sequel, we will prove that $x(t)$ is a positive solution.

Firstly, notice that $x(t)$ is a fixed point of the operator $(T u)(t)=\int_{0}^{1} G(t, s) f(s, u(s)) \mathrm{d} s$ and, consequently,

$$
x(t)=\int_{0}^{1} G(t, s) f(s, x(s)) \mathrm{d} s .
$$

Now, suppose that there exists $0<t^{*}<1$ such that $x\left(t^{*}\right)=0$. This means that

$$
x\left(t^{*}\right)=\int_{0}^{1} G\left(t^{*}, s\right) f(s, x(s)) \mathrm{d} s=0 .
$$

Using that $x(t)$ is a nonnegative function, $f(t, y)$ is nondecreasing with respect to the second argument and the nonnegative character of $G(t, s)$, we get

$$
0=x\left(t^{*}\right)=\int_{0}^{1} G\left(t^{*}, s\right) f(s, x(s)) \mathrm{d} s \geq \int_{0}^{1} G\left(t^{*}, s\right) f(s, 0) \mathrm{d} s \geq 0 .
$$

This gives us $x\left(t^{*}\right)=\int_{0}^{1} G\left(t^{*}, s\right) f(s, 0) \mathrm{d} s=0$.

As $G(t, s) \geq 0$ and $f(s, 0) \geq 0$, the last expression implies

$$
G\left(t^{*}, s\right) f(s, 0)=0 \quad \text { a.e }(s) .
$$

As $G\left(t^{*}, s\right) \neq 0$ a.e $(s)$ (because $G\left(t^{*}, s\right)$ is given by a polynomial), we can obtain

$$
f(s, 0)=0 \quad \text { a.e }(s) \text {. }
$$

On the other hand, as $f\left(t_{0}, 0\right) \neq 0$ for certain $t_{0} \in[0,1]$, the nonnegative character of $f(t, y)$ gives us $f\left(t_{0}, 0\right)>0$. As $f(t, y)$ is a continuous function, we can find a set $A \subset[0$, 1] with $t_{0} \in A, \mu(A)>0$, where $\mu$ is the Lebesgue measure and $f(t, 0)>0$ for any $t \in$ $A$. This contradicts (6).

Therefore, $x(t)>0$ for $t \in(0,1)$. This finishes the proof.

Remark 3. In Theorem 4, the condition $f\left(t_{0}, 0\right) \neq 0$ for certain $t_{0} \in[0,1]$ seems to be a strong condition in order to obtain a positive solution for Problem (2), but when the solution is unique, we will see that this condition is very adjusted one. In fact, suppose that Problem (2) has a unique nonnegative solution $x(t)$ then

$$
f(t, 0)=0 \text { for each } t \in[0,1] \text { if and only if } x(t) \equiv 0 .
$$

In fact, if $f(t, 0)=0$ for each $t \in[0,1]$, it is easily seen that the zero function satisfies Problem (2) and the uniqueness of the solution gives us $x(t)=0$. The reverse implication is obvious. 
Remark 4 . Notice that the hypotheses in Theorem 3 are invariant by continuous perturbation. More precisely, if $f(t, 0)=0$ for any $t \in[0,1]$ and $f$ satisfies (H1) and (H2) of Theorem 3 then $g(t, x)=a(t)+f(t, x)$ with $a:[0,1] \rightarrow[0, \infty)$ continuous and $a \neq$ 0 , satisfies assumptions of Theorem 4 , and this means that the following boundary value problem

$$
\left.\begin{array}{l}
D_{0^{+}}^{\alpha} u(t)+g(t, u(t))=0, \quad 0<t<1 \\
u(0)=u(1)=u^{\prime}(0)=0
\end{array}\right\}
$$

has a unique positive solution.

Now, we present an example that illustrates our results.

Example 1. Consider the boundary value problem

$$
\left.\begin{array}{c}
D_{0^{+}}^{\frac{5}{2}} u(t)+c+\lambda \cdot \operatorname{arctg} u(t)=0, \quad 0<t<1, c, \lambda>0 \\
u(0)=u(1)=u^{\prime}(0)=0
\end{array}\right\}
$$

In this case, $\alpha=\frac{5}{2}$ and $f(t, u)=c+\lambda \cdot \operatorname{arctg} u$. It is easily seen that $f(t, u)$ satisfies (H1) of Theorem 3.

In the sequel, we prove that $f(t, u)$ satisfies (H2) of Theorem 3.

Previously, we consider the function $\varphi:[0, \infty) \rightarrow[0, \infty)$ given by $\varphi(u)=\operatorname{arctg} u$ and we will see that $\varphi$ satisfies

$$
\phi(u)-\phi(v) \leq \phi(u-v), \quad \text { for } u \geq v .
$$

In fact, put $\varphi(u)=\operatorname{arctag} u=\alpha$ and $\varphi(v)=\operatorname{arctg} v=\beta$ (notice that, as $u \geq v$ and $\varphi$ is nondecreasing, $\alpha \geq \beta$ ).

Then, from

$$
\operatorname{tg}(\alpha-\beta)=\frac{\operatorname{tg} \alpha-\operatorname{tg} \beta}{1+\operatorname{tg} \alpha \cdot \operatorname{tg} \beta}
$$

and, as $\alpha, \beta \in\left[0, \frac{\pi}{2}\right)$, then $\operatorname{tg} \alpha, \operatorname{tg} \beta \in[0, \infty)$, we can obtain

$$
\operatorname{tg}(\alpha-\beta) \leq \operatorname{tg} \alpha-\operatorname{tg} \beta .
$$

Applying $\varphi$ to the last inequality and taking into account the nondecreasing character of $\varphi$, we obtain

$$
\alpha-\beta \leq \operatorname{arctg}(\operatorname{tg} \alpha-\operatorname{tg} \beta),
$$

or, equivalently,

$$
\phi(u)-\phi(v)=\operatorname{arctg} u-\operatorname{arctg} v=\alpha-\beta \leq \operatorname{arctg}(u-v)=\phi(u-v) .
$$

This proof our previous claim.

Now, for $u \geq v$ and $t \in[0,1]$, we have,

$$
f(t, u)-f(t, v)=\lambda(\operatorname{arctg} u-\operatorname{arctg} v) \leq \lambda \operatorname{arctg}(u-v) .
$$

Now, we prove that $\varphi(u)=\operatorname{arctg} u$ belongs to $\mathcal{J}$. Obviously, $\varphi:[0, \infty) \rightarrow[0, \infty)$ is a continuous and nondecreasing function. Moreover, $\psi(u)=u-\varphi(u)=u-\operatorname{arctg} u$ is also continuous and nondecreasing and satisfies $\psi(u)>0$ for $u>0$ and $\psi(0)=0$. Consequently, $\phi \in \mathcal{J}$. 
Finally, as $f(t, 0)=c+\operatorname{arctg} 0=c>0$, by Theorem 4, Problem (7) has a unique positive solution for

$$
0<\lambda \leq\left(\frac{1}{\Gamma(5 / 2+1)}\left[\left(\frac{3}{5}\right)^{3 / 2}-\left(\frac{3}{5}\right)^{5 / 2}\right]\right)^{-1} \approx 17.8682 .
$$

\section{Some remarks}

In a recent paper [18], the authors study the existence of positive solutions of a particular case of Problem (2). More precisely, they study the following fractional autonomous boundary value problem

$$
\begin{aligned}
& D_{0^{+}}^{\alpha} u(t)+\lambda f(u(t))=0, \quad 0<t<1 \\
& u(0)=u(1)=u^{\prime}(0)=0,
\end{aligned}
$$

where $2<\alpha \leq 3, \lambda$ is a positive parameter and $f:(0, \infty) \rightarrow(0, \infty)$ is continuous. The main tool used by the authors in this paper is Guo-Kranosel'skii fixed-point theorem on cones. In [18], the question about the uniqueness of solutions is not treated.

One of the results of [18] is the following theorem.

Theorem 5. [[18], Theorem 3.2] If there exists $l \in(0,1)$ such that $q(l) c_{2} f_{0}>F_{\infty} c_{1}$ holds then, for each $\lambda \in\left(\left(q(l) c_{2} f_{0}\right)^{-1},\left(F_{\infty} c_{1}\right)^{-1}\right)$, the boundary value problem (8) has at least one positive solution.

Here, we consider $\left(q(l) c_{2} f_{0}\right)^{-1}=0$ if $f_{0}=\infty$ and $\left(F_{\infty} c_{1}\right)^{-1}=\infty$ if $F_{\infty}=0$, where $F_{\infty}=\lim _{u \rightarrow+\infty} \sup \frac{f(u)}{u}, F_{\infty}=\lim _{u \rightarrow+\infty} \sup \frac{f(u)}{u}, q(t)=t^{\alpha-1}(1-t), k(s)=s(1-s)^{\alpha-1}$, $c_{1}=\frac{1}{\Gamma(\alpha)} \int_{0}^{1}(\alpha-1) k(s) \mathrm{d} s$, and $c_{2}=\frac{1}{\Gamma(\alpha)} \int_{0}^{1} \frac{1}{\alpha-1} q(s) k(s) \mathrm{d} s$.

Now, we present the following example.

Example 2. Consider the boundary value problem that is a variant of Example 1.

$$
\begin{aligned}
& D_{0^{+}}^{5 / 2} u(t)+\lambda(c+\operatorname{arctg} u(t))=0, \quad 0<t<1, c, \lambda>0, \\
& u(0)=u(1)=u^{\prime}(0)=0,
\end{aligned}
$$

In this case, $\alpha=\frac{5}{2}$ and $f(u)=c+\operatorname{arctg} u$. Then, we have $F_{\infty}=0$ and $f_{0}=\infty$. Moreover, $c_{1}=0.129, c_{2}=0.0077$, and $q(1 / 2)=\frac{\sqrt{2}}{8}=0.1768$ [[18], Example 5.1]. Thus, $q$ $(1 / 2) c_{2} f_{0}>F_{\infty} c_{1}$ holds. Theorem 5 gives us the existence of a positive solution for Problem (9) for each $\lambda \in(0, \infty)$. The question of uniqueness cannot be treated by the results of [18].

On the other hand, following a similar reasoning that in Example 1, Theorem 4 gives us the existence of a unique positive solution for Problem (9) when $0<\lambda \leq\left(\frac{1}{\Gamma(5 / 2+1)}\left[\left(\frac{3}{5}\right)^{3 / 2}-\left(\frac{3}{5}\right)^{5 / 2}\right]\right)^{-1} \approx 17.8682$.

Our main contribution is the uniqueness of positive solution for Problem (9) when 0 $<\lambda \leq 17.8682$.

Now, we present an example that cannot be studied by the results of [18], and it can be treated by the ones obtained in this paper.

Example 3. Consider the following boundary value problem

$$
\begin{aligned}
& D_{0^{+}}^{5 / 2} u(t)+\lambda(t+\operatorname{arctg} u(t))=0, \quad 0<t<1, \lambda>0, \\
& u(0)=u(1)=u^{\prime}(0)=0,
\end{aligned}
$$


In this case, the boundary value problem is nonautonomous, and thus, this problem cannot be studied by the results of [18].

On the other hand, using a similar argument that in example 1, and using Theorem 4, we obtain the existence of a unique positive solution for Problem (10) when $0<\lambda \leq$ 17.868 .

\section{Acknowledgements}

This research was partially supported by "Ministerio de Educación y Ciencia" Project MTM 2007/65706.

\section{Authors' contributions}

We are part of the same research group and work together therefore, we can affirm that the contents of this paper has been prepared by all the authors: JC, JH, and KS. All authors read and approved the final manuscript.

\section{Competing interests}

The authors declare that they have no competing interests.

Received: 28 February 2011 Accepted: 18 September 2011 Published: 18 September 2011

\section{References}

1. Diethelm, K, Freed, AD: On the solutions of nonlinear fractional order differential equations used in the modelling of viscoplasticity. In: Keil F, Mackens W, Voss H, Werthers J (eds.) Scientific Computing in Chemical Engineering IIComputational Fluid Dynamics, Reaction Engineering and Molecular Properties. pp. 217-224. Springer, Heidelberg (1999)

2. Gaul, L, Klein, P, Kempffe, S: Damping description involving fractional operators. Mech Syst Signal Process. 5, 81-88 (1991). doi:10.1016/0888-3270(91)90016-X

3. Glockle, WG, Nonnenmacher, TF: A fractional calculus approach of self-similar protein dynamics. Biophys J. 68, 46-53 (1995). doi:10.1016/S0006-3495(95)80157-8

4. Mainardi, F: Fractional calculus: Some basic problems in continuum and statistical mechanics. In: Carpinteri CA, Mainardi F (eds.) Fractal and Fractional Calculus in Continuum Mechanics. pp. 291-348. Springer, Wien (1997)

5. Metzler, F, Schick, W, Kilian, HG, Nonnenmache, TF: Relaxation in filled polymers: A fractional calculus approach. J Chem Phys. 103, 7180-7186 (1995). doi:10.1063/1.470346

6. Oldham, KB, Spanier, J: The Fractional Calculus. Academic Press, New York (1974)

7. Kilbas, AA, Trujillo, JJ: Differential equations of fractional order: Methods, results and problems-I. Appl Anal. 78, 153-192 (2001). doi:10.1080/00036810108840931

8. Samko, SG, Marichev, OI, Kilbas, AA: Fractional Integral and Derivative, Theory and Applications. Gordon and Breach, Yverdon, Switzerland (1993)

9. Miller, KS, Ross, B: An Introduction to the Fractional Calculus and Fractional Differential Equations. Wiley, New York (1993)

10. Podlubny, I: Fractional Differential Equations. Academic Press, San Diego (1999)

11. Lakshmikantham, V, Vatsala, AS: Basic theory of fractional differential equations. Nonlinear Anal. 69, 2677-2682 (2008). doi:10.1016/j.na.2007.08.042

12. Lakshmikantham, V: Theory of fractional functional differential equations. Nonlinear Anal. 69, 3337-3343 (2008). doi:10.1016/j.na.2007.09.025

13. Bai, C: Positive solutions for nonlinear fractional differential equations with coefficient that changes sign. Nonlinear Anal. 64, 677-685 (2006). doi:10.1016/j.na.2005.04.047

14. Bai, Z, Ge, W: Existence of three positive solutions for some second-order boundary value problems. Comput Math Appl. 48, 699-707 (2004). doi:10.1016/j.camwa.2004.03.002

15. Bai, Z, Lü, H: Positive solutions for boundary value problem of nonlinear fractional differential equation. J Math Anal Appl. 311, 495-505 (2005). doi:10.1016/j.jmaa.2005.02.052

16. Bai, Z: On positive solutions of a nonlocal fractional boundary value problem. Nonlinear Anal. 72, 916-924 (2010). doi:10.1016/j.na.2009.07.033

17. Zhang, S: Existence of solution for a boundary value problem of fractional order. Acta Math Sci. 26, $220-228$ (2006)

18. Zhao, Y, Sun, S, Han, Z, Li, Q: Positive solutions to boundary value problems of nonlinear fractional differential equations. Abs Appl Anal. 2011, Article ID390543 (2011)

19. Harjani, J, Sadarangani, K: Fixed point theorems for weakly contractive mappings in partially ordered sets. Nonlinear Anal. 71, 3403-3410 (2009). doi:10.1016/j.na.2009.01.240

20. Kilbas, AA, Srivastava, HM, Trujillo, Jj: Theory and Applications of Fractional Differential Equations. In North-Holland Mathematics Studies, vol. 204,Elsevier, Amsterdam (2006)

21. Yu, Y, Jiang, D: Multiple positive solutions for the boundary value problem of a nonlinear fractional differential equation. Northeast Normal University, Changchun, Jilin Province, Republic of China PRC (2009)

22. Nieto, JJ, Rodríguez-López, R: Contractive mapping theorems in partially ordered sets and applications to ordinary differential equations. Order. 22, 223-239 (2005). doi:10.1007/s11083-005-9018-5

23. Harjani, J, López, B, Sadarangani, K: On positive solutions of a nonlinear fourth order boundary value problem via a fixed point theorem in ordered sets. Dyn Syst Appl. 19, 625-634 (2010)

doi:10.1186/1687-2770-2011-25

Cite this article as: Caballero et al:: On existence and uniqueness of positive solutions to a class of fractional boundary value problems. Boundary Value Problems 2011 2011:25. 